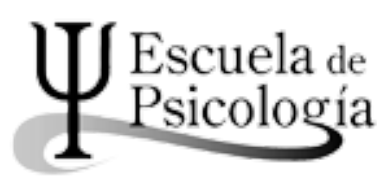

Wímblu, Rev. estudiantes Esc. de psicología, Univ. de Costa Rica. 12 (1): 85-96, 2017 / ISSN: 1659-2107

\title{
Espacios que habitar: \\ Memorias de la organización de estudiantes de Psicología, Sede de Occidente de la UCR, 2012
}

\author{
Spaces to live: Remembrances of psychology students organization, \\ Occidental campus University of Costa Rica, 2012
}

Marco Rojas Lizano*

Resumen: Este texto versa sobre las memorias de un grupo de estudiantes de la carrera de Psicología en la Sede de Occidente de la Universidad de Costa Rica, en torno a la organización realizada para conseguir el presupuesto y así mantener la presencia de la carrera no solo en la Sede, sino también en la Región de Occidente.

Palabras clave: Memorias, Organización estudiantil-Psicología Sede de Occidente.

\begin{abstract}
This text describes the remembrances of a group of students from the Psychology career of the University of Costa Rica Occidental Campus, about the organization that was performed to obtain budget in order to maintain the career active not only in the campus but also in the Occidental Region.
\end{abstract}

Key Words: Remembrances, students Organization, Psychology at the Occicental Campus.

Universidad de Costa Rica. Bachiller en Psicología. Sede de Occidente. Costa Rica. Correo electrónico: marco.rojaslizano@ucr.ac.cr

Recepción: 15/11/2016 Aceptación: 20/2/2017 


\section{A manera de introducción}

No se podría iniciar un acercamiento a la realidad experimentada por quienes, de alguna manera, estuvimos vinculados a la carrera de Psicología en la Sede de Occidente de la Universidad de Costa Rica (UCR) en el año 2012, sin detenerse antes a pensar en las condiciones históricas, sociales, económicas y políticas que han demarcado parámetros para el devenir de la universidad pública, al menos en el marco latinoamericano.

La universidad pública en Latinoamérica esta ubicada en medio de tensiones que, por un lado, apuntan a desarrollar prácticas educativas de calidad e inclusión con un compromiso ético y social, mientras que por otro lado, existen intentos de la mercantilización y transnacionalización de esta educación, demandas de un sistema económico neoliberal que de conducen a un quiebre del compromiso con los sectores excluidos a quienes debería aspirar a trabajar (Martínez y Sánchez, 2014).

La Sede de Occidente, como por lógica podrá deducir quien lee, no escapa de estas tensiones, por el contrario reflejo de lo anterior, se ha podido observar cómo ante problemáticas relacionadas con limitaciones presupuestarias, inmediatamente remiten los recortes a las carreras del área de ciencias sociales.

Es en el medio de esta coyuntura, que para el año 2012 decisiones administrativas de la Sede llevan a un intento por recortar el presupuesto designado para las carreras de Psicología y Enseñanza de la Música. Este posicionamiento conduce al a organización de un grupo de estudiantes de psicología, apoyados por un músculo estudiantil representante de otras carreras, a realizar una serie de acciones reivindicando el impacto y la importancia que hasta ese momento habían logrado tener no solo en la Sede de la universidad, ubicada en San Ramón, sino además para el resto de la Región de Occidente, mediante el aporte de los y las estudiantes a través prácticas de cursos, trabajos comunitarios y otras vinculaciones (para el año 2012 aún no se realizaba ningún Trabajo Final de Graduación).

El presente texto tiene por intensión presentar las memorias de una serie de personas, estudiantes, vinculadas con la organización estudiantil para mantener la presencia de la carrera de Psicología en la Sede. A estas participantes, todas mujeres (dos entrevistas individuales (Entrevistas 1 y 4 ) dos entrevistas grupales (a tres compañeras: entrevista 2; a dos compañeras: emtrevista 3)), se suman una serie de evocaciones propias, pues en ese momento formé parte de dicha movilización. Parto del entendimiento de que en el intento de reconstruir una serie de memorias, en lugar de tomar una distancia desde una supuesta crítica que advierta cierta objetividad, me ubico desde una participación emotiva (Rossi, 2003, citado por Dobles, 2009).

Esto me remite a la pregunta que intuyo en la lectura de Alvarado, De Ríos y Nova (2016): ¿cómo adquiera actualidad la memoria? Siguiendo a estos autores, parto de que la memoria es un lugar que se debe habitar y que este acto genera la posibilidad de configurar un mundo-espacio que apela por una presencia y participación de nosotras y nosotros como sus actores.

Encontré en mis reflexiones y lecturas que escribir este texto solo desde mis memorias no tendría mucho sentido, pues de cualquier modo estas siempre apelan a su configuración a razón de un grupo, en este caso el de organización estudiantil. Además, siguiendo a Dobles (2009, p. 41) apunto que la memoria es una construcción colectiva o social en tanto "se articula en las relaciones sociales y se compone mediante proceso y prácticas sociales que las definen".

Es claro, el intento de que este texto llegue a otros espacios más allá de mi computadora no es un acto inocente o de mero repaso de fechas. No, por el contrario responde a los intentos de actuales estudiantes de la carrera por conmemorar las posibilidades de organización y acción de los grupos dentro de estos centros educativos y cómo, en el caso específico de la carrera de psicología, apuestan por una permanencia en ese espacio físico que se materializa en el cantón de San Ramón pero que se expande y alcanza otros como Grecia, Sarchí, Naranjo, Palmares y Zarcero.

Además, este es un pequeño esfuerzo por hacer frente a las posturas ahistóricas, individualizantes, naturalizadores de las lógicas de dominación, buscando, como argumentara Martín-Baró (1991), devolver al ser humano a su historia. Es este caso visibilizar el contexto y estructuras en las que se vieron insertas parte de las vivencias de quienes en aquel momento, no muy lejano, decidieron organizarse, no dejar las cosas por dadas y mantener la carrera de Psicología en la Región de Occidente.

\section{De rumores a información}

Hacia inicios del segundo semestre del año en cuestión, se genera una alerta entre estudiantes, la cual remite a la posibilidad de un recorte de presupuesto para la carreras de Psicología y de Enseñanza de la Música, que al ser las más recientemente creadas, se encontraban en una particular condición de vulnerabilidad.

En el caso de la carrera de Psicología, desde la administración de la Sede, se había incitado a que de manera conjunta, como suele suceder, se realizara la feria vocacional, la cual convoca a distintos centros de enseñanza secundaria, 
principalmente ubicados en la zona de acción de la Sede, en esa tan pensada Región de Occidente. Bajo esta premisa, estudiantes quienes se habían visto preocupados por la posible situación de la carrera, la cual era comunicada a través de rumores, encuentran un espacio de tranquilidad pues, como argumentan "para qué hacer feria vocacional si no se iba a abrir [la nueva promoción]"(Entrevista 2 UCR.SO, 2015, 1). La realización de dicha feria llegaría funcionar como un mecanismo que mermó un poco la incipiente organización estudiantil.

Dicha lógica va a ser cuestionada un tiempo después, cuando a partir de comunicaciones entre la coordinación de la carrera, cuerpo docente y estudiantes, se confirme que existe un recorte presupuestario, que se traduciría en la falta de tiempos docentes para el año 2013, lo cual significaría la no apertura de una nueva promoción de Psicología en la Sede de Occidente.

Con esto se inician una serie de nuevos rumores entre estudiantes, basados en la ambigua información que se manejaba. Se escuchaba entre pasillos y en las aulas, que presupuesto había, sin embargo que en el Concejo de Sede, se tomó la decisión de no apoyar más presupuestariamente a la carrera. A esto se le suma la preocupación a partir del conocimiento de la experiencia y la tan reciente herida del cierre de la carrera en Limón, la cual se había encontrado con una situación similar que al final significó para muchas personas la búsqueda de cupo en la Sede Rodrigo Facio, el desplazamiento que esto conlleva y posteriormente como conoceríamos años más tarde, para quienes se quedaron, la imposibilidad para encontrar docentes que se quisiesen sumar como parte de equipos asesores para los trabajos finales de graduación.

En el marco de este escenario, se empiezan a dar las primeras reuniones informativas, las cuales fueron integradas por pocas personas, principalmente aquellas miembros de la Asociación de Estudiantes de Psicología de la Sede (AEPSO) y algunas que se habían acercado para tener información de primera mano, tratando de entender y dimensionar las implicaciones del cierre de la promoción que tenía para nosotros y nosotras como estudiantes. En una de estas reuniones se toma la decisión de emitir una carta a la coordinadora de la carrera en ese momento, Fabiola Villalobos, con la cual pedíamos que se nos diese toda la información necesaria e invitábamos al cuerpo docente a elaborar de una serie de estrategias para mantener la carrera en la región. Dicha carta está fechada el 2 de octubre del 2012.

Con estas primeras informaciones que pasaron de ser un rumor a confirmarse con datos provenientes de la coordinación de la carrera, se empieza a detonar una movilización de las y los estudiantes, que va más allá de los órganos legitimados en los espacios estudiantiles, como en este caso es la AEPSO y la Asociación de Estudiantes de la Sede de Occidente (AESO), sino que se expandió a una organización con diferentes niveles de participación y compromiso, un espacio que tuvo la intensión de articularse a manera de asamblea, en donde cada quien asumiría responsabilidades según su posibilidad. Dichas movilizaciones pasaron por distintos lugares, pero en este inicio al menos, su lugar fue la necesidad de hacer frente a un proceso del cual, la coordinación ya no podía hacer más. Una de las entrevistadas recuerda:

muchos sentíamos que la parte administrativa [coordinación] decía que ya habían hecho todo lo posible verdad, entonces que ya nos tocaba a nosotros y a la vez era como unas ganas de luchar pero a la vez como sentirse un poco desarmado de no saber hacia donde, pero que ahí entre todos nos dábamos como fuerza, recuerdo mucho...(Entrevista 3 UCR.SO, 2015, 1).

Ya teníamos la información y existía una incipiente organización estudiantil movida por elementos individuales, como lo podría ser el hecho de perder un curso y al existir años sin promoción, tener que esperar para poder volver a llevarlo (situación mencionada en varias de las entrevistas) pero principalmente por elementos que pasan por la solidaridad y la construcción de continuar el espacio en una región con necesidades a las cuales, dicha carrera en su espacio de trabajo con las comunidades, podría colaborar de alguna manera. A partir de esto se generaron las primeras acciones.

\section{Primeras acciones}

"A la misma vez empezó a darse algo muy interesante de que entre los diferentes niveles empezamos a comunicarnos" (Entrevista 1 UCR.SO, 2015, 1)

No podría hablar con propiedad de las experiencias en otras sedes, pues solo cursé psicología en la de Occidente, salvo un par de cursos de último nivel, pero esta cuenta con la peculiaridad de que las relaciones entre pares parecen encontrar una delimitación en los grupos de cada generación. Para el momento del 2012 la comunicación entre los distintos niveles era escasa.

Con la notificación de las dificultades presupuestarias, la necesidad de comunicar lo que algunas personas sabían se hizo evidente. Las reuniones informativas empezaron a ser más frecuentes en los almuerzos de las semanas, en donde mientras se comía a la vez se discutían nuestras posibles acciones y consecuencias en los peores escenarios (Entrevista 3 UCR.SO, 2015). Una de las entrevistadas recuerda que se empezó por hacer una contextualización de las dinámicas a nivel nacional e internacional referentes a la educación superior, pues se partía que para poder generar un proceso de organización estudiantil, se necesitaban bases sólidas que demarcaran la ruta a transitar (Entrevista 1 UCR. SO, 2015). Además se repasaba y se buscaba información con personas de la carrera en Limón, quienes nos compartieron su experiencia y señalaron posibles 
acciones a realizar. Esta entrevistada recuerda además que dichas reuniones permitieron aclarar el terreno en el que nos movilizaríamos, tratando también de generar una motivación entre quienes participaron de ellas (Entrevista 1 UCR.SO, 2015). A partir de estas primeras reuniones se empezaron a aportar ideas para una vez siendo reflexionadas, pasar a la acción.

Una de las primeras acciones como organización, fue solicitar información clara y justificada al director de la Sede, en ese momento Francisco Rodríguez, acerca de la decisión que se había tomado en el Concejo, del porqué no abrir la promoción de psicología del 2013. Dicha carta está fechada el día 23 de octubre de 2012. Esta fue contestada el 29 del mismo mes refiriéndonos a la carta que había sido enviada a la coordinación, sin más respuesta, sin más explicación que el acuse a problemas presupuestarios.

Se seguía discutiendo acerca de la situación por la que estábamos pasando, sin embargo se consideró que se había hablado mucho, que era momento de empezar a tomar acciones. Lo primero, unirnos como carrera.

\section{La Vigilia}

Enunciada desde varios lugares tales como vigilia, lunada, entre otros, esta fue la noche en la que se convocaron a estudiantes de psicología principalmente, pero a la cual también se sumaron en solidaridad personas de la carrera de Artes Plásticas, Trabajo Social, Derecho y además de otras que integraban en ese momento la AESO. La consigna era clara y sencilla: empezar a visibilizar nuestra organización. Esta fue dada por quienes en ese entonces se encontraban en los últimos cursos de licenciatura, es decir la primer generación que ingresó a la carrera en la Sede.

El punto de encuentro fue la fuente, ubicada frente a la Biblioteca Arturo Agüero Chávez. Esto tuvo la intensión de ser una acción hacia afuera, es decir, hacia el resto de la comunidad estudiantil que probablemente no conocía la situación, sin embargo esta vigilia se convirtió en un espacio para compartir entre quienes ya integraban la organización. Una de las compañeras entrevistadas lo recuerda así: "lo que se hizo fue como más actividades que nos integraron: se pintó, se pusieron carteles, se pintó el piso ahí, estábamos en la fuente" ( Entrevista 2 UCR. SO, 2015:p. 4 ) . En realidad, mucho pasó por lo lúdico en una primera instancia, juegos, música y dejar esa señal, ese signo, que apropiándose de la tipografía del No al TLC2 ${ }^{2}$ dejó con tizas lo que se leía como No al cierre de Psico.

Posteriormente, en lo que se podría pensar como una segunda etapa de esa vigilia, guardas de seguridad nos comunicaron que no podíamos permanecer en las instalaciones más allá del cierre de portones, el cual se daría a las 10 pm. Sin embargo llegamos a un acuerdo a la hora de explicarles nuestros motivos, por lo que nos trasladamos a la entrada de la Sala Rosada de la biblioteca. En esta se dio a una asamblea en la cual se discutieron los siguientes pasos y acciones para lograr el objetivo. Era una noche muy fría, de cuyas fotos se pueden recordar personas con mantas, cobijas, bolsas de dormir.

...luego se dio el espacio de discusión sobre lo que estaba pasando y sobre que hacer. Luego de ahí, nos querían sacar entonces nos fuimos a sentar afuera de la sala rosada y ahi se hizo como un memorandum o algo asi, se escribio en pape periódico los pasos a seguir a partir de ese día (Entrevista 2 UCR.SO, 2015, p.4).

Con esta narración, la compañera menciona la construcción colectiva y posterior votación de aspectos que consideramos necesarios para poder articular de una mejor manera todos los esfuerzos para tener un más amplio margen de acción. Todas estas ideas quedaron plasmadas en un cartel el cual no permanecería estático, se tacharían cosas, se cambiarían sus propuestas a partir de la forma en que acontecerían esos breves días.

Como balance de esa noche, concuerdo con todas las personas entrevistadas, en que un principio no sabíamos como serviría esa vigilia para visibilizarnos (la Sede cuenta con la particularidad que después de las cinco de la tarde, es muy poca la gente que asiste a cursos), sin embargo permitió acoplar una serie de iniciativas, que si bien tenían la intensión de buscar estrategias para que la carrera permaneciese en la Región, no todas se habían compartido para el colectivo de estudiantes. De esta manera lo expresa una de las entrevistadas:

...sí, ¿que se hizo? Creo que primero fue, bueno, es que la idea, creo que la idea de la actividad en sí era para unificarnos, y para que se hablara de la problemática y la sede, pero no era algo como de fuese a dar resultados inmediatos sino era como para que nosotros lográramos organizarnos (Entrevista 2 UCR.SO, 2015, p.3).

Era la primer actividad convocada a la que asistía una representación importante de las cuatro generaciones que en ese momento integraban la carrera. De aquí en adelante las acciones realizadas seguirían estando integradas por un significativo número de estudiantes, principalmente de psicología, pero acompañadas por otras personas de diferentes carreras. 


\section{El Pasacalles}

“....si se pudiese hacer una radiografía de los afectos hubiera estado. no se hubieran visto nunca las partes negras, todo hubiera sido blanco porque era muy fuerte lo que estábamos viviendo" (Entrevista 1 UCR.SO, 2015, p.4)

Fueron varias las propuestas planteadas el día de la vigilia y con una manera fácil de llegar al consenso. Dentro de estas opciones, se planteó realizar una actividad, ahora sí, en un horario en el que la sede estuviese llena de estudiantes, una actividad que no solo se hiciera escuchar la voz de la organización, sino también una acción, directa que dejara huella.

Nunca existió una línea establecida, en realidad como dice una las personas entrevistadas,"si había alguien deseando hacer algo salido de las normas, ese era el momento" (Entrevista 1 UCR.SO, 2015,p.4). Justamente el pasacalles es mencionado en las entrevistas con palabras y frases como canciones, caras pintadas, además de los lugares transitados, como lo fueron el auditorio, los laboratorios de matemática y biología, Vida Estudiantil-Registro, la fuente, los pabellones de aulas, el comedor, la dirección (para el 2012, por cuestiones de remodelación, la Dirección de la Sede se encontraba ubicada contigual comedor y la cancha, en el mismo espacio de Transporte). Lo que se hizo fue: que yo tenía en la mente, verdad, entonces se hizo como un desfile, un pasacalles por toda la universidad, empezaron abajo, creo en el auditorio pasaron todo hasta arriba, tocaban las puertas de las aulas, verdad, para que se unieran, explicando ah muchos de nosotros (Entrevista 3 UCR.SO, 2015, p.4)

Se empezó por el auditorio, ubicado en el primer bloque de edificios de la Sede, en el sector este. Empezaron las canciones, creadas justamente para este día, se subieron las gradas que abundan en este espacio, se pasó por las aulas explicando la situación e invitando a sumarse a quienes estaban en estas clases, a apoyar la organización. Estudiantes de Trabajo Social y Artes Plásticas, consecuentes con su compromiso no se hicieron esperar. Compañeras de la organización se descalzaron y con los pies pintados de ocre fueron dejando huella por toda la sede.

Yo creo que hubo creatividad en las propuestas y eso también ayudo muchísimo, un pasacalles con colores con la posibilidad de hacer ciertas cosas que irrumpían en espacio con zapatos pintados en las aceras, también era eso, digamos, dejar una huella en la u y en el espacio físico también llamaba a participar, entonces eso, eso, creo que también convocaba a la gente (Entrevista 1 UCR.SO, 2015, p.4).
Se culmina la actividad en la Dirección de la Sede. Por primera vez desde que se emitiera la carta comunicando a la coordinación de la carrera la decisión tomada por el Concejo de Sede de cerrar la promoción 2013, el director atiende a alguien relativo a Psicología, en este caso, a toda la organización estudiantil.

"llegamos a la dirección y el...ya estaba afuera porque ya había escuchado el escandalo. Ahi como que lo hicimos comprometerse, bueno, le preguntamos que era lo que estaba pasando especificamente con Psico, que porque era que la querían cerrar, entonces el ya nos explico que no habian tiempos y no sé qué, entonces que habia que escoger dos carreras que se quedaran por fuera y que se habia decidido que psicología y que no era como nada personal y que la carrera como era la más nueva...Y entonces me acuerdo que le preguntaron que si nosotros lográbamos conseguir los tiempos que si el se comprometía de palabra a aprobarlos, abrir las nuevas promociones de música y de psicología. Y hasta lo estaba grabando y todo y el mae que sí, que si nos aprobaban la plata el se comprometía con abri como que esa fue la discusión ahí central (Entrevista 2 UCR.SO, 2015, p. 5-6).

Como lo narra la compañera, uno los primeros pasos se estaba logrando, conseguir aunque fuese de palabra un compromiso de parte de uno de los integrantes del Concejo de Sede y además director del recinto, para que nos diera libre paso para avanzar al siguiente nivel, el cual consistía conseguir los tiempos docentes en la Vicerrectoria de Docencia.

Inmediatamente terminado el pasacalles, algunas personas se dirigieron al espacio de la AESO para redactar una carta en la cual se le solicitaba la revocatoria de lo indicado en el oficio SO-DCS-CPS-052-2012 de la sesión ordinaria No 882 la cual tomaba la decisión del cierre de la promoción de la carrera para el 2013.

El pasacalles, además de conseguir que el director de la Sede nos atendiera, sirvió para consolidar esa unidad y fuerza de la organización estudiantil, una de las acciones estructurantes como estudiantes de psicología:

el pasacalles tuvo mayor alcance, en el sentido de lo que estamos hablando de que no sé, formó grupalidad, eh, en su momento sí verdad... entonces en ese sentido creo que fue muy estructurante y memorable, yo creo que si en este momento le pregunta a cualquiera yo creo que le va a decir el pasacalles... (Entrevista 1 UCR. SO, 2015, p. 5).

Me parece necesario rescatar que dentro de las entrevistas se valoriza las posibilidades de efectivamente lograr los objetivos planteados desde la organización de los y las estudiantes, en medio de un contexto que trata de vaciar de sentido todas aquellas formas que se posicionen desde una visión crítica y que apunten a un compromiso para con el otro y la otra.

...me parece que ayudó demasiado a formar un espíritu de lucha que ya nos hacía sí complices a muchos en el grupo..... a mi en lo personal me hizo pensar en [que] 
que vaya en contra del sistema, me hizo pensar en ciertos contextos, en ciertos momentos, se pueden articular luchas que den sus frutos... ( Entrevista 1 UCR.SO

Con el pasacalles se consiguió abrir el portillo para que la administración de la Sede se comprometiera a que si se conseguían los tiempos docentes, estos serían dados a las carreras que se estaban viendo excluidas del presupuesto.

\section{A manera de cierre}

Se dieron más acciones, como por ejemplo un plantón en una Asamblea de Sede, de la cual nunca voy a olvidar a un profesor de filosofía diciendo que no se sentía seguro con estudiantes en las entradas del auditorio. También una visita al Concejo Universitario en la que fuimos recibidos por el rector y el vicerrector de docencia en la cual nos comunicaban que se nos otorgaban los tiempos docentes. Sin embargo, rescato en este breve texto los puntos anteriores, aquellas acciones que en realidad lograron estructurar a un grupo de estudiantes para desarrollar una propuesta: lograr que se mantenga la presencia de la Carrera de Psicología desde la Sede en la Región de Occidente.

Alrededor de cuarenta años atrás, Martín-Baró (1975) proponía que como parte de las labores y compromiso de los y las estudiantes universitarios, está la ruptura con los supuestos de que la participación estudiantil solo debe darse en ciertas áreas, las legitimadas desde lugares hegemónicos de control, las que les encasilla en una supuesta postura apolítica. En la Sede, más allá de presenciar pasivamente como desde ciertos lugares se tomaba la decisión de comprometer su espacio de formación y acción, los y las estudiantes se articularon en el proponer y hacer. Es por esto que a un grupo de personas nos parece importante el compartir estas memorias.

La memoria se reconstruye más allá de recuperarse (Halbwachs, 1947 citado en Dobles, 2009), articulando bajo este precepto cierta noción que da espacio a una inventiva o creatividad que apunte a la utilización de estas memorias como mecanismo de acción en el presente. De esta manera con Alvarado, de los Ríos y Nova (2016) podríamos decir que resistir tiene que ver con habitar creativamente la memoria. A la hora de habitar esta memoria, desde este caso específico, hay un antes y un después de la organización de estudiantes en el 2012:

...yo estaba pensando en eso en estos días y pensando en esa pregunta [interrupción] yo creo que en las generaciones que estuvimos ahí, vinculadas con ese proceso, hay que hasta ese momento en ningún momento habían mostrado ningún interés por que tipo de cosas, y a partir de ese momento fueron otros, entonces creo interes po identitario fueron demasiado importantes (Entrevista 1 UCR. SO, 2015: 3).
...Digamos, yo recuerdo que ese periodo de tiempo y me gusta mucho recordarlo. Osea, a pesar de que era como de incertidumbre y de lucha digamos, creo que era tuvimos porque todas las generaciones unimos, y eran cuatro, obviamente siempre hay gente que se queda por fuera de cada generación, que no se involucra por hay gente que se queda por fuera de cada generacion, que no se involucra por de cuando veo las fotos... porque es demasiado lindo que estábamos todos juntos, todos una pelota, todos nos movíamos al mismo ritmo e intentábamos de aportar con lo que fuera... ( Entrevista 2 UCR.SO, 2015, p. 8)

Como forma de aproximarme a la pregunta que planteaba al inicio del texto, encuentro que el habitar estas memorias responde a un acto político en tanto estas experiencias permitan la articulación de acciones en el presente, como una forma de enfrentarlo.

Para concluir quisiera decir que este texto es solo una invitación a otras personas a analizar lo vivido por este grupo de estudiantes en el 2012 y que a la vez pueda ser compartido en escenarios que apuntan a tensionar los procesos de educación y formación profesional. De igual manera quisiera apostar por la construcción de una propuesta ético política de la Psicología, que basándose en la en Dussel (1998) y en las discusiones que sobre este hacen Flores (2014) y Dobles (2016), apele a producir y reproducir la vida humana en nuestros pueblos a partir de la revisión crítica de nuestro quehacer cotidiano tanto dentro como fuera de las aulas.

\section{Notas}

1. Entrevista de Joaquín Soler a Carlos Fuentes, 1977. Recuperado desde https://www.youtube. $\mathrm{com} /$ watch?v=e8DUO0gbo58

2. La tipografía de la campaña del Movimiento Patriótico No al TLC, aludía con su letra o a un corazón, como estrategia de incluir al cuerpo $y$ a las emociones en el espacio político, un corazon, como estrategia de incluir al cuerpo y a las emociones en el espacio político,
del cual históricamente han sido relegadas. Para más, revisar el texto de Mércedes Álvarez, "Movimientos sociales y participación política: movimieno contra el TLC en la campaña de "Movimientos sociales y participación politica: movimieno contra el TLC en la campaña 2007 en Costa Rica, Anuario de Estudios Centroamericanos 37, (2011): 207.

\section{Referencias}

Alvarado, Victor; De los Ríos, Alicia; Nova, Mayra. (2016). Habitar la memoria en América Latina. De contar la historia a encender el fuego de nuevo. Pacarina del Sur Revista de Pensamiento Crítico Latinoamericano, (28), 224-259. Recuperado desdehttp://pacarinadelsur.com/58-dossiers/dossier-18/1334habitar-la-memoria-en-latinoamerica-de-contar-la-historia-a-encender-elfuego-nuevo 
Álvarez, Mercedes. (2011). Movimientos sociales y participación política: el movimientocontra el TLC en la campaña del referendum 2007 en Costa Rica. Anuario de Estudios Centroamericanos, 37, 201-230. Recuperado desde http://www.jstor.org.ezproxy.sibdi.ucr.ac.cr:2048/stable/pdf/41306439.pdf

Dobles, Ignacio. (2009). Memorias del dolor. Consideraciones acerca de las Comisiones de la verdad en América Latina. San José: Editorial Arlekin, 2009.

Dobles, Ignacio. (2016). Ignacio Martín-Baró. Una lectura en tiempos de quiebres y esperanzas. San José: Editorial Arlekín.

Dussel, Enrique. (1998). Ética de la Liberación en la edad de la globalización y de la exclusión. Madrid: Editorial Trotta.

Flores, Jorge. (2014). Psicología y ética comunitaria. En Flores, Jorge (Coord), Pensar la Psicología y lo Comunitario en América Latina. Tijuana: Centro Latinoamericano de Investigación, Intervención y Atención Psicosocial.

Martín-Baró, Ignacio. (1991). El método en psicología política".Suplementos Anthropos, (40), 30-39.

Martínez, Gloriana; Sánchez, Adriana. (2014). Nuevas Voces en Ciencias Sociales: una mirada a la investigación social. Reflexiones, 93 (1), 179-178. Recuperado desde revistas.ucr.ac.cr/index.php/reflexiones/article/download/13752/1308

\section{Fuentes primarias}

Entrevista a estudiante de Psicología UCR.SO 1, San Pedro. (2015).

Entrevista a estudiantes de Psicología UCR.SO 2, Naranjo. (2015).

Entrevista a estudiantes de Psicología UCR.SO 3, San Ramón. (2015).

Entrevista a estudiante de Psicología UCR.SO 4, San Ramón. (2015).

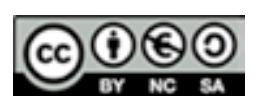

Esta obra está bajo una licencia de Creative Commons Reconocimiento

- NoComercial - CompartirIgual (by-nc-sa) 